\title{
Phase boundary dynamics of bubble flow in a thick liquid metal layer under an applied magnetic field
}

\author{
Mihails Birjukovs $\odot,{ }^{*}$ Valters Dzelme, and Andris Jakovics \\ Institute of Numerical Modelling, University of Latvia, UL, Jelgavas iela 3, LV-1004 Riga, Latvia \\ Knud Thomsen $\odot$ and Pavel Trtik ${ }^{\dagger}$ \\ Paul Scherrer Institut, PSI, Forschungsstrasse 111, 5232 Villigen, Switzerland
}

(Received 2 November 2019; accepted 27 May 2020; published 18 June 2020)

\begin{abstract}
We investigate argon bubble flow in liquid gallium within a container large enough to avoid wall effects. Flow with and without applied horizontal magnetic field is studied. We demonstrate the successful capture and quantification of the effects of applied magnetic field using dynamic neutron radiography and the previously developed and validated robust image processing pipeline, supported by the in silico reproduction of our experiment. Significant reduction of the amplitude of bubble tilt angle variations due to applied horizontal magnetic field is successfully resolved through a $30 \mathrm{~mm}$ thick liquid metal layer. Our results clearly show the potential of expanding the range of gas/liquid metal systems that can be studied using downscaled though representative experimental setups.
\end{abstract}

DOI: 10.1103/PhysRevFluids.5.061601

Multiphase flow in the presence of external magnetic field (MF) is of great fundamental and practical interest. Many metallurgical processes, such as melt stirring, homogenization, purification [1], as well as liquid metal column chemical reactors [2], involve gas (oftentimes inert gas such as argon, Ar) flow through electrically conductive fluids, controlled and stabilized by applied MF [1,39]. To optimize these processes, the underlying physics must be well established. Aside from the fact that many aspects of single gas bubble hydrodynamics, let alone bubble collective dynamics and interactions with MF (i.e., coalescence, splitting, collisions, agglomeration, phase boundary/wake dynamics), are not fully understood [1,3,10-16], liquid metal systems are notoriously difficult to probe noninvasively, due to their opacity, high temperatures and additional complications due to strong MF [1]. Consequently, visual data regarding how applied MF alters bubble shape and bubble collective dynamics are scarce.

The issue with high temperatures and large scales of industrial hardware can be sidestepped by using smaller volumes of low melting point model liquids, such as gallium $(\mathrm{Ga})$ or galinstan (GaInSn), provided the actual system's dimensionless parameters are sufficiently close to model system's [1]. However, to extract physical information from experiments, appropriate experimental and data processing methods must enable tracking the bubbles, resolving their boundaries, and capturing dynamics thereof.

There is currently a profound lack of a robust approach, whereas rising to this challenge would yield fundamental insights into complex interactions between fluid flow, unsteady bubble shapes, and MF, and thus unlock new verification criteria, which should be as direct as possible, for validation of rapidly developing computational fluid dynamics (CFD) models.

\footnotetext{
*mihails.birjukovs@lu.lv

†pavel.trtik@psi.ch
} 
Several velocimetry methods of varied reliability for liquid metal systems exist, such as contactless inductive flow tomography (CIFT) $[17,18]$ and ultrasound Doppler velocimetry (UDV) $[1,3,19]$, but these only yield liquid metal velocity field and simply reveal flow perturbations from which bubble presence may be inferred.

With optical methods removed form consideration due to liquid metal opacity to visible light, the ultrasound transit time technique (UTTT), x-ray radiography (XR), x-ray computed tomography (XCT), and neutron radiography (NR) remain among the viable methods.

UTTT is very limited in that it only detects the general location and characteristic size of a bubble within metal, and yields no information about its shape, meaning that surface perturbations, being unresolved, may also introduce errors in position and velocity estimates [3,6-8].

$\mathrm{XR}$ and XCT methods allow for direct observation of bubble shapes. However, $\mathrm{x}$-ray based techniques are, in general, very constrained by relatively small liquid metal thickness in the beam direction due to intense X-ray attenuation by liquid metals [20-24]. XCT, while offering very high temporal resolution and sufficient phase boundary detection precision, also entails experimental systems that are rather susceptible to applied MF, rendering them hardly applicable to studies of magnetohydrodynamic (MHD) bubble flow [25-27].

$\mathrm{NR}$, on the other hand, while similar in principle to XR, allows, for some metals, probing much thicker samples and, as such, is a very promising experimental method that should enable broader coverage of the parameter spaces of various liquid metal systems $[2,28,29]$. While considerable progress has been made outside of the liquid metal context and without MF [30-32], only a few advances with some notable exceptions, have been achieved for liquid metal systems using NR, and to our knowledge it has not been used to systematically study the MF effects on bubble flow $[2,28,29,33-36]$.

The successful capture of the dynamics of rising bubbles requires image acquisition with high temporal resolution $(\sim 10 \mathrm{~ms})$. With the fluxes available even at the high-performance neutron sources, such short image acquisitions lead to a rather low signal-to-noise ratio (SNR). Previously, we have demonstrated bubble flow dynamics sufficiently resolved by a uniquely tailored processing pipeline designed for low SNR neutron images. Applied horizontal MF (HMF) was seen to stabilize bubble trajectories, damping lateral velocity and displacement, and resulting in nearly straight ascension paths, as opposed to zigzag motion observed without MF. Image-based velocimetry revealed bubble acceleration in the upper half of the melt container in the presence of MF, while the opposite is seen without applied MF [37]. Hereinafter, we demonstrate that our approach also resolves phase boundary dynamics, not just translational motion.

Experiments were conducted at the thermal neutron imaging beamline NEUTRA at the Paul Scherrer Institute (PSI) [38]. The setup (Fig. 1), a detailed description of which is found in [37], consisted of a $150 \times 90 \times 30 \mathrm{~mm}^{3}$ glass container filled with liquid $\mathrm{Ga}$, wherein Ar bubble chain flow was introduced via a submerged copper tube with a constricted nozzle, which ejected bubbles such that they ascended without wall interactions. This was necessary to isolate and study the bubble-bubble interactions and MF effects.

It is important to note that the $30 \mathrm{~mm}$ thickness is a highlight. With this, we aim to push the boundaries of feasible (in terms of NR) thickness of a scaled down system, improving the coverage within a system's parameter space. Previous experiments with liquid metals by other groups featured $25 \mathrm{~mm}$ thick rectangular containers [2]; a pipe with a $24 \mathrm{~mm}$ diameter and a flat $10 \mathrm{~mm}$ thick vessel (studied flow regimes different from what is considered here and did not perform bubble velocimetry and shape/trajectory analysis) $[35,36]$. It should also be noted that the effects of a horizontal/vertical MF were not studied in $[2,35,36]$. This means that the sample thickness in the setup presented herein is, to our knowledge, currently the highest in its class.

To study the influence of applied HMF, the container was placed between two arrays of neodymium permanent magnets. MF flux density within the container was $\sim 300 \mathrm{mT}$ within the bubble flow region, as determined by simulations and measurements. Simulations indicated that MF at the inlet was $\vec{B} \sim(100 ; 17 ;-3.5) \mathrm{mT}$. The direction of the field was rather homogeneous along the container vertical axis and exhibited a moderate gradient in the vertical direction. 

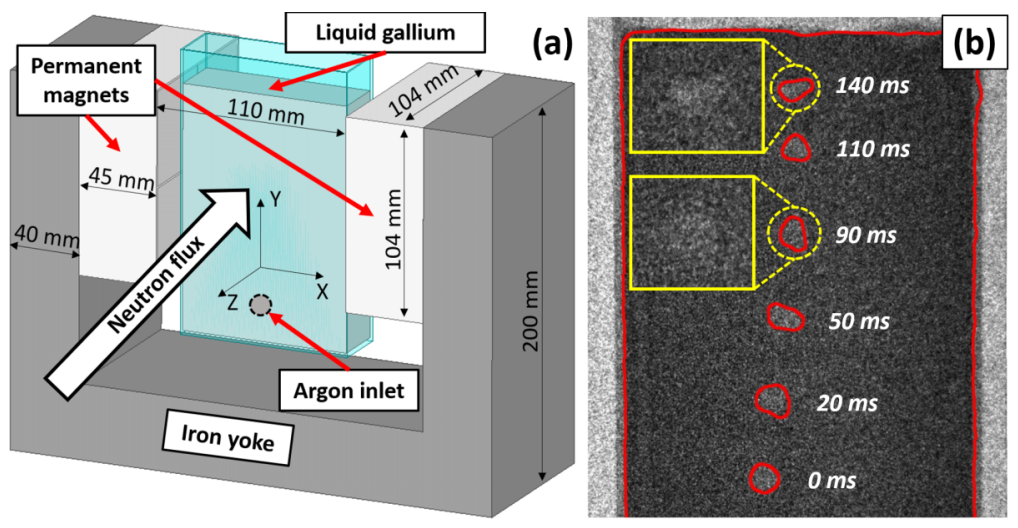

FIG. 1. (a) Experimental setup with highlighted components, dimensions, and neutron flux direction. (b) A composition of several sequential flat-field and dark current corrected neutron images overlaid with the detected container walls, air/Ga, and $\mathrm{Ar} / \mathrm{Ga}$ phase boundaries. In (b) close-ups of bubbles and the background noise are shown (yellow frames).

The experimental setup was imaged at 100 frames per second (10 ms exposure time) for $100 \mathrm{~cm}^{3} / \mathrm{min}$ Ar flow rate, with and without applied MF. Neutron images were dark current and flat-field corrected, denoised using the self-snakes type curvature flow filter, then bubble shapes were extracted via double-Otsu morphological binarization and Shen-Castan edge detection with bubble interior and edge restoration subroutines. Bubble shape parameters were derived from best-fit ellipses [37].

The experiment was reproduced numerically to verify that observed effects are not artifacts. Bubble dynamics with applied MF in the volume of fluid (VOF) formulation are governed by the following system of dimensionless equations:

$$
\begin{aligned}
& \rho \frac{d \vec{v}}{d t}=-\underbrace{\nabla p}_{\begin{array}{c}
\text { Pressure } \\
\text { gradient }
\end{array}}+\underbrace{\frac{\nabla \cdot \tau}{\operatorname{Re}}}_{\begin{array}{c}
\text { Viscous } \\
\text { forces }
\end{array}}+\underbrace{\frac{\rho \vec{g}}{\mathrm{Fr}^{2}}}_{\text {Buoyancy }}+\underbrace{\frac{\rho k}{\text { Eo }} \nabla \alpha}_{\text {Surface tension }} \\
& +\underbrace{N \sigma[-\nabla \phi+\vec{v} \times(\nabla \times \vec{A})] \times(\nabla \times \vec{A})}_{\text {Lorentz force }}, \\
& \underbrace{\Delta \vec{A}}_{\begin{array}{c}
\text { Field } \\
\text { diffusion }
\end{array}}=-\underbrace{\mathrm{Rm} \cdot \sigma[-\nabla \phi+\vec{v} \times(\nabla \times \vec{A})]}_{\text {Induced current }}-\underbrace{\nabla \times \vec{B}_{r}}_{\begin{array}{c}
\text { Permanent } \\
\text { magnets }
\end{array}}, \\
& \frac{\partial \alpha}{\partial t}+\vec{u} \cdot \nabla \alpha=0, \\
& \nabla \cdot(\rho \vec{v})=0, \\
& \nabla \cdot(\sigma \nabla \phi)=\underbrace{\nabla \cdot[\sigma \vec{v} \times(\nabla \times \vec{A})]}_{\text {Induced potential }},
\end{aligned}
$$

where all entities are dimensionless and $\vec{A}$ is the magnetic vector potential, $\phi$ is the electric potential, $\sigma$ is the VOF electric conductivity, $\vec{v}$ is the velocity field, $\vec{B}_{r}$ is the remanent MF flux density due to the permanent magnets, $\rho$ is the VOF density, $p$ is pressure, $\tau=\mu\left(\nabla \vec{v}+(\nabla \vec{v})^{T}\right)$ is the Newtonian viscous stress tensor, $\mu$ is the VOF dynamic viscosity, $\vec{g}$ is the gravitational acceleration, $k$ is the 
phase boundary curvature (given by $k=\nabla \vec{n}$, where $\vec{n}=\nabla \alpha /|\nabla \alpha|$ ), and $\alpha$ is the volume fraction of $\mathrm{Ga}(\alpha \in[0 ; 1])$.

$\sigma, \rho$, and $\mu$ are given by VOF blending functions of the form $X=\alpha+(1-\alpha) X_{\mathrm{Ar}} / X_{\mathrm{Ga}}$, where $X$ is a fluid property. The flow regime is prescribed by the magnetic Reynolds number $\mathrm{Rm}=\mu_{0} \sigma_{0} U L$, the hydrodynamic Reynolds number $\operatorname{Re}=U L / v_{0}$, the Froude number $\operatorname{Fr}=U / \sqrt{g_{0} L}$, the Stuart number $N=\sigma_{0} B_{0}^{2} L / \rho_{0} U$, and the Eötvös number Eo $=\delta \rho \cdot g_{0} L^{2} / \gamma$, where $U$ and $L$ are velocity and length scales, respectively, $\mu_{0}$ is the vacuum permeability, $\nu_{0}$ is the scale for the kinematic viscosity, $g_{0}$ is the gravitational acceleration modulus, $\rho_{0}$ is the density scale, $\gamma$ is the surface tension, and $\delta \rho$ is the $\mathrm{Ar} / \mathrm{Ga}$ density difference. Equation (2) gives the dynamics of applied MF perturbed by currents induced due to bubble flow and Eq. (1) governs the velocity field within the container, driven by body forces. Volume fraction transport is controlled by (3), where phase boundaries correspond to $\Delta \alpha=0$. Equations (4) and (5) are the continuity equations for the velocity and the current density fields, respectively.

Material parameters used in the CFD models and the tools used to solve Eqs. (1)-(5) numerically are found in [37]. Preliminary analysis of our setup, images, and simulations show that one has $\mathrm{Fr} \in[0.2 ; 0.3]$, implying that buoyancy is the main driving force, as expected. $\mathrm{Rm} \in[0.01 ; 0.1]$, so MF due to current induced by metal flow through the applied MF could be pronounced. Estimates indicate that Eo $\in[2.1 ; 4.1](6-8 \mathrm{~mm}$ sized bubbles) and the Reynolds number near bubbles is within $\operatorname{Re} \in\left[10^{3} ; 10^{4}\right]$, which corresponds to a flow regime wherein bubbles are of oscillating elliptic shapes $[13,39]$.

At the same time, $\operatorname{Re} \sim 1-10$ elsewhere, so an appropriate turbulence model must be used to resolve shape dynamics and trajectories. The $k$-equation subgrid-scale (SGS) large eddy simulation (LES) model was chosen to avoid artificial bubble trajectory stabilization due to overestimated turbulent viscosity, which we find is introduced by a more standard $k-\omega$ shear stress transport (SST) model.

$N \in[0.8 ; 3.5]$ near bubbles, indicating that the Lorentz force should strongly affect metal flow. It is known that, for these values of the Stuart number, MF, horizontal or vertical (or both components, if the field in not homogeneous), radically changes the structure of bubble wake and bubble trajectory characteristics [3-5].

Turning to our experiment and simulations, the main results are presented in Fig. 2, wherein bubble tilt angle dynamics averaged over $30 \mathrm{~s}$ of bubble flow recordings for $100 \mathrm{~cm}^{3} / \mathrm{min}$ flow rate are shown with and without applied HMF, as well as respective simulation output averaged over $1 \mathrm{~s}$ of flow time. For a $30 \mathrm{~s}$ neutron image recording, $\sim 2000-2500$ bubble detection events over the entire usable field of view (FOV) $(8-10 \mathrm{~cm}$ height depending on the noise in the image sequence) contribute to the statistics. We define the tilt angle $\phi$ as the angle between the major semiaxis of the best-fit ellipse for a bubble and the $X$-axis (the horizontal dimension of the images); this is illustrated in Fig. 2(a).

In Fig. 2(a), notice that the $\phi$ magnitude in the case with no applied field has a maximum of roughly $40^{\circ}$ for both experimental and simulated curves, as opposed to an initial maximum of $20^{\circ}$ with applied MF, were one also observes subsequent near zero $\phi$ for CFD data and greatly reduced amplitude, but with more noise (lower SNR) for experimental data at higher elevations, as seen in Fig. 2(b); the difference is obvious from Fig. 2(c).

To interpret these results, consider that without applied MF and for low Re the wake of a solitary rising bubble is axisymmetric and stable [1,11]. As Re increases, the symmetry is broken and then, as the pair of hairpin-shaped tail vortices on each side of the bubble (with respect to the major semiaxis plane) increases in size further due to do progressively more detached boundary layer, vortex shedding ensues $[1,4,5,10,11]$. This occurs even for stable bubble shapes but is further enhanced by and in turn also affects surface perturbations and asphericity [4,5,10]. As a result of vortex shedding, nonzero net lift force is generated beneath the bubble in its wake, forcing it to deflect significantly, which then transitions to periodic lateral motion in alternating directions, as more vortices are shed and higher/lower pressure regions alternate beneath the bubble. 

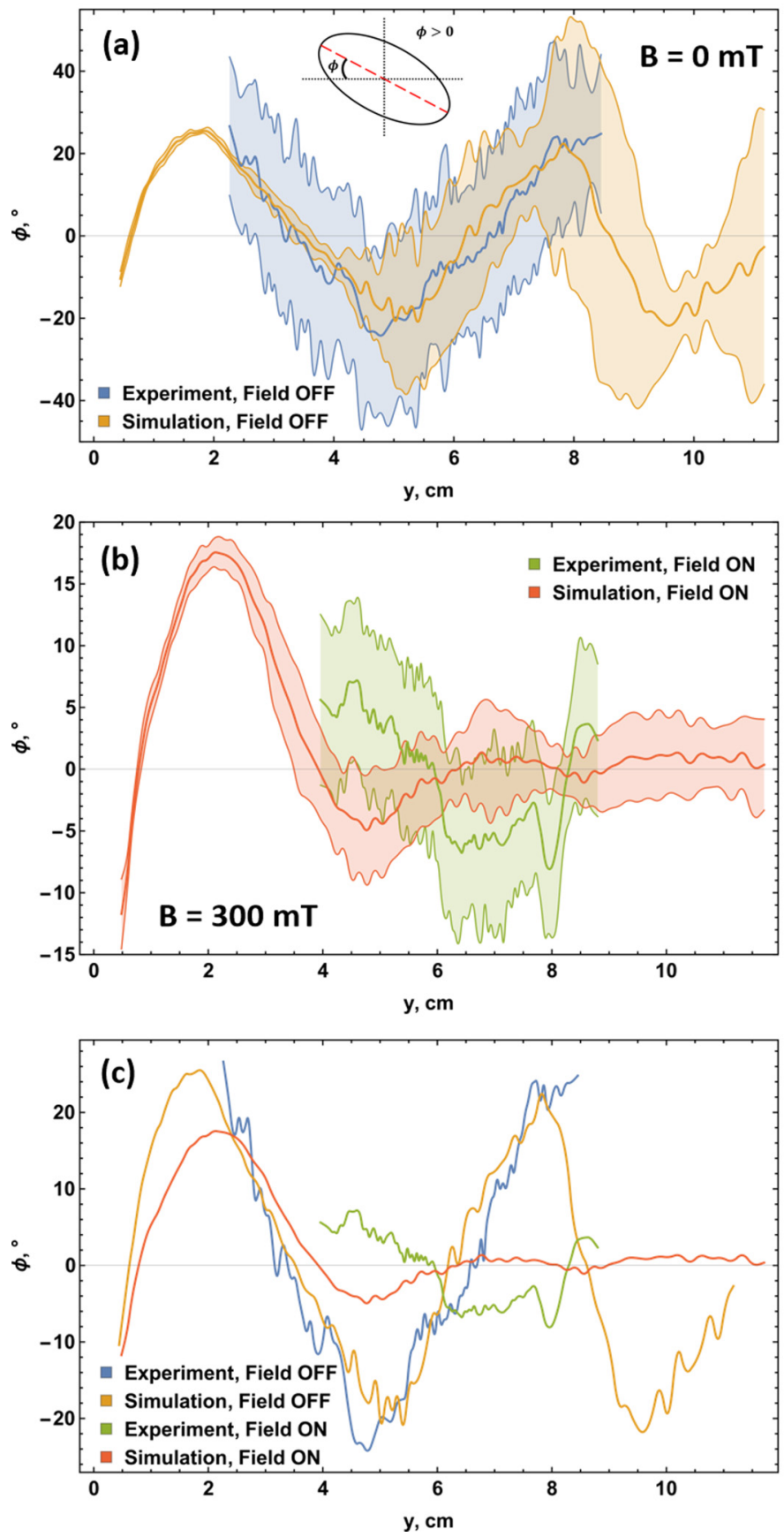

FIG. 2. Experimentally observed and simulated tilt angle and bubble elevation height correlations (a) without and (b) with applied HMF with color bands representing local errors for averaged curves; (c) shows a direct comparison of averaged tilt curves with and without applied field. Flow rate is at $100 \mathrm{~cm}^{3} / \mathrm{min}$. The positive tilt angle is defined as shown in (a).

Bubbles with Re and Eo ranges considered herein exhibit very unstable and complex turbulent wake structures enhanced by significant shape deformations $[13,39]$, which is well illustrated in 


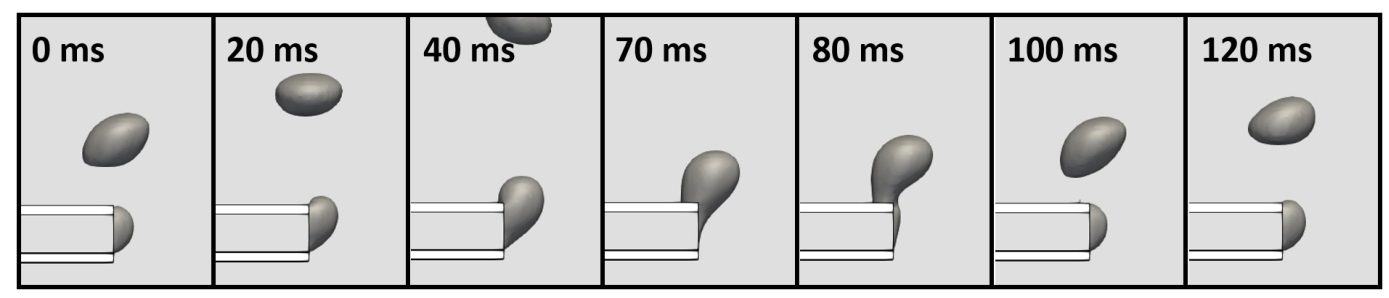

FIG. 3. Simulated bubble (dark gray) detachment at the inlet (white).

[5] (Fig. 30). Bubble paths are expected to be zigzags, with chaotic out-of-plane deviations due to shape instabilities and turbulent pulsations within the wake, accompanied by periodic bubble tilting $[5,10,11]$.

This correlates very well with the results obtained without applied MF, as seen in Fig. 2, where, in addition, numerical predictions match experimental findings very well within the radiography FOV for which our image processing pipeline reliably resolves phase boundaries. In Fig. 2(a), note that initially the error band of the simulated curve is much narrower. During bubble formation at the horizontally directed (and thus asymmetric) inlet tube [37], the bubble is stretched vertically by the buoyancy forces and laterally due to hydrodynamic gas pressure at the tube opening; then, once the bubble detaches, surface tension forces retract the lagging part of the bubble towards its center of mass, resulting in self-propulsion and rapid initial acceleration responsible for the starting negative $\phi$ values. This is illustrated in Fig. 3.

Then, violent vortex detachment occurs, resulting in rapid change of sign of $\phi$, after which the bubble proceeds to ascend normally. The reason for the initially low dispersion of the $\phi$ values is that in this initial interval the effects of self-propulsion and the first vortex detachment dominate over bubble-bubble interactions. For a chain of rising bubbles (here the characteristic bubble-bubble distance is $\sim 3-4$ bubble diameters), as the elevation increases, continued vortex shedding and trailing bubble interactions with wakes from leading ones result in chaotic bubble deflections and tilt angle variations, explaining the increased width of the error band, which then coincides with that of the experimental results. However, this initially narrow trajectory corridor is below the data noise threshold.

When HMF is applied, depending on the $N$ value, one observes the recovery of a rectilinear path $[3,6]$, which in the case of data shown in Fig. 2(b) translates to an initial interval with significant deflection and $\phi$, followed by ascension with greatly reduced $\phi$. The initially higher $\phi$ is due to the same mechanisms as in the case of no field. However, note that in Fig. 2(b) the maximum $\phi$ within the initial region is slightly less when MF is applied. This and the later low $\phi$ regime are observed because in liquid metal with high $\sigma$ such as $\mathrm{Ga}$, flow about the rising bubble induces electric currents near the bubble and within its wake. These currents interact with the applied MF, resulting in the Lorentz force which opposes and damps fluid flow in the direction depending on the MF orientation (other velocity components are altered indirectly via the continuity constraint), as illustrated in Fig. 4 [1]. In our case, magnetic damping is mostly due to the transverse field (TF) alignment [orthogonal to the bubble centroid velocity, Fig. 4(a)], but one must also consider the effects of the partial longitudinal field (LF) alignment [Fig. 4(b)], which occurs during bubble formation and right after its detachment at the inlet tube (Fig. 3).

TF damping directly affects the velocity components orthogonal to the applied field, which here is the vertical component. Other components are, in turn, affected due to flow velocity field continuity. As seen in [5] (Fig. 30), applied TF results in several effects. At moderate flux density, the finer wake structure disappears and vortices are elongated vertically. This can be attributed to flow velocity decrease leading to wake laminarization. Also note the decreased width of the bubble wake stemming from the vortex size diminished by TF damping. However, the wake is still asymmetric. At higher TF flux density, damping is intense enough to reduce the effective bubble Re value below 

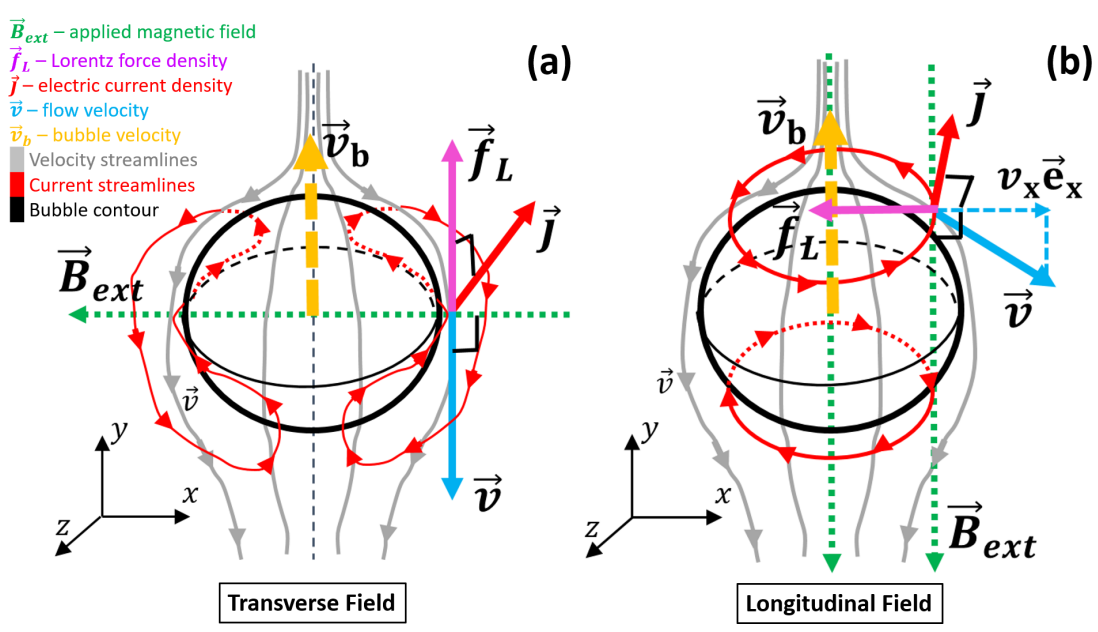

(b)

FIG. 4. Idealized representations of the effects of magnetic damping of flow near a bubble for (a) transverse and (b) longitudinal field alignment. Note that in (b) in the lower hemisphere the $v_{x}$ sign is inverted and so is the direction of the Lorentz force density vector.

the vortex shedding threshold, resulting in stabilized pairs of counter-rotating vortices and greatly decreased wake length, and thus also a reduced bubble-bubble interaction range. At this point, rectilinear trajectory is recovered, since lift force asymmetry is no longer sufficient to noticeably defect the bubble. LF damping, on the other hand, directly affects the horizontal velocity component and other components are altered due to the continuity constraint. It has been demonstrated that this results in effects slightly different from the TF case, but also stabilizes bubble trajectory [4,7].

Actual physics will clearly deviate from this idealized picture, but this simplified treatment may allow for interpretation of our results. Qualitatively, it makes sense that during bubble formation and takeoff from the inlet, due to its asymmetry, both LF and TF mechanisms are triggered, reducing lateral deflection and tilt. As the flow around the bubble is damped and laminarized, the trajectory stabilizes and becomes nearly rectilinear (note also that the MF flux increases with elevation, as shown in [37]), with the magnetic TF (tilt magnitude is very small) damping maintaining a stable, symmetrized wake until the bubble reaches the surface. Despite minor discrepancies between the experimental and numerical results with applied MF [Figs. 2(b) and 2(c)], it is evident that in both cases the $\phi$ magnitude is dramatically decreased and both the experiment and the numerical model indicate $\phi$ values that agree rather well, especially given the low image SNR.

To summarize, we have demonstrated the bubble shape parameter variations captured via dynamic NR. The bubble shape parameters were successfully extracted from inherently low SNR images, while the investigated liquid metal layers were substantially thick (i.e., representative of industrial applications). As such, our approach shows potential to significantly expand the range of gas/liquid metal systems that can be studied using experimental setups downscaled via similarity criteria (Re, Rm, Eo, $N$, etc.). Furthermore, we have reproduced our experiment in silico and have verified our experimental findings by comparing the recovered bubble tilt angle dynamics to the numerical results. We have found that applying static HMF decreases bubble tilt angle oscillation magnitude dramatically, an observation concurrent with our CFD simulations and contemporary physical knowledge within the field. While we still aim to improve our experimental setup and the image processing pipeline, and numerical and experimental studies spanning the Re, Eo, and $N$ parameter space involving vertical/horizontal MF are necessary, it is clear that NR is a feasible, robust, and reliable tool for in-depth bubble shape dynamics studies in liquid metals, especially when combined with appropriate post-processing methods. Our experimental results agree well with simulations and literature, implying that our methodology can correctly resolve rapidly changing 
liquid/gas phase boundaries, paving the way to further studies of the physics of MHD bubble flow in liquid metal and elucidation of exactly how MF orientation and magnitude affect bubble surface shape stability and collective dynamics. The presented approach offers perspectives with details from phase boundary velocimetry for many systems of interest, with the potential to be used for direct verification of numerical models and codes.

This work is based on the experiments performed at the Swiss spallation neutron source SINQ, Paul Scherrer Institute (PSI). The authors are grateful to Jevgenijs Telicko [University of Latvia (UL), Latvia] and Jan Hovind (PSI, Switzerland) for their invaluable assistance with hardware during experiments, Robert Zboray (Empa Dübendorf, Switzerland) for productive discussions regarding data post-processing, and Martins Klevs (UL, Latvia) for assistance with numerical simulations. The authors also express gratitude to the anonymous referees for many helpful comments. The authors acknowledge the support due to the ERDF project "Development of numerical modelling approaches to study complex multiphysical interactions in electromagnetic liquid metal technologies" (No. 1.1.1.1/18/A/108).

[1] C. Zhang, Liquid metal flows driven by gas bubbles in a static magnetic field, Dissertation, Technischen Universität Dresden, 2009, https://d-nb.info/1008503541/34.

[2] E. Baake, T. Fehling, D. Musaeva, and T. Steinberg, Neutron radiography for visualization of liquid metal processes: bubbly flow for $\mathrm{CO}_{2}$ free production of hydrogen and solidification processes in EM field, IOP Conf. Ser.: Mater. Sci. Eng. 228, 012026 (2017).

[3] E. Strumpf, Experimental study on rise velocities of single bubbles in liquid metal under the influence of strong horizontal magnetic fields in a flat vessel, Int. J. Multiphase Flow 97, 168 (2017).

[4] S. Schwarz and J. Fröhlich, Numerical study of single bubble motion in liquid metal exposed to a longitudinal magnetic field, Int. J. Multiphase Flow 62, 134 (2014).

[5] K. Jin, P. Kumar, S. Vanka, and B. Thomas, Rise of an argon bubble in liquid steel in the presence of a transverse magnetic field, Phys. Fluids 28, 093301 (2016).

[6] T. Richter, O. Keplinger, N. Shevchenko, T. Wondrak, K. Eckert, S. Eckert, and S. Odenbach, Single bubble rise in GaInSn in a horizontal magnetic field, Int. J. Multiphase Flow 104, 32 (2018).

[7] C. Zhang, S. Eckert, and G. Gerbeth, Experimental study of single bubble motion in a liquid metal column exposed to a DC magnetic field, Int. J. Multiphase Flow 31, 824 (2005).

[8] Z. Wang, S. Wang, X. Meng, and M. Ni, UDV measurements of single bubble rising in a liquid metal galinstan with a transverse magnetic field, Int. J. Multiphase Flow 94, 201 (2017).

[9] J. Zhang, M.-J. Ni, and R. Moreau, Rising motion of a single bubble through a liquid metal in the presence of a horizontal magnetic field, Phys. Fluids 28, 032101 (2016).

[10] G. Mougin and J. Magnaudet, Path Instability of a Rising Bubble, Phys. Rev. Lett. 88, 014502 (2001).

[11] W. L. Shew, S. Poncet, and J.-F. Pinton, Force measurements on rising bubbles, J. Fluid Mech. 569, 51 (2006).

[12] T. Ziegenhein and D. Lucas, Observations on bubble shapes in bubble columns under different flow conditions, Exp. Therm. Fluid Sci. 85, 248 (2017).

[13] M. Tripathi, K. Sahu, and R. Govindarajan, Dynamics of an initially spherical bubble rising in quiescent liquid, Nat. Commun. 6, 6268 (2015).

[14] K. Wichterle, M. Večeř, and M. Růžička, Asymmetric deformation of bubble shape: Cause or effect of vortex-shedding? Chem. Papers 68, 74 (2014).

[15] B. Bunner and G. Tryggvason, Effect of bubble deformation on the properties of bubbly flows, J. Fluid Mech. 495, 77 (2003).

[16] A. Loisy, A. Naso, and P. Spelt, Buoyancy-driven bubbly flows: Ordered and free rise at small and intermediate volume fraction, J. Fluid Mech. 816, 94 (2017). 
[17] F. Stefani, T. Gundrum, and G. Gerbeth, Contactless inductive flow tomography, Phys. Rev. E 70, 056306 (2004).

[18] T. Gundrum, G. Gerbeth, F. Stefani, and M. Xu, Experimental aspects of contactless inductive flow tomography, Magnetohydrodynamics 42, 153 (2006).

[19] T. Vogt, A. Andruszkiewicz, S. Eckert, K. Eckert, S. Odenbach, and G. Gerbeth, Mixing enhancement in gas-stirred melts by rotating magnetic fields, Metall. Mater. Trans. B 43, 1454 (2012).

[20] O. Keplinger, N. Shevchenko, and S. Eckert, Validation of X-ray radiography for characterization of gas bubbles in liquid metals, IOP Conf. Ser.: Mater. Sci. Eng. 228, 012009 (2017).

[21] L. Liu, O. Keplinger, T. Ziegenhein, N. Shevchenko, S. Eckert, H. Yan, and D. Lucas, Euler-Euler modeling and $\mathrm{X}$-ray measurement of oscillating bubble chain in liquid metals, Int. J. Multiphase Flow 110, 218 (2018).

[22] B. Krull, E. Strumpf, O. Keplinger, N. Shevchenko, J. Fröhlich, S. Eckert, and G. Gerbeth, Combined experimental and numerical analysis of a bubbly liquid metal flow, IOP Conf. Ser.: Mater. Sci. Eng. 228, 012006 (2017).

[23] O. Keplinger, N. Shevchenko, and S. Eckert, Experimental investigation of bubble breakup in bubble chains rising in a liquid metal, Int. J. Multiphase Flow 116, 39 (2019).

[24] O. Keplinger, N. Shevchenko, and S. Eckert, Visualization of bubble coalescence in bubble chains rising in a liquid metal, Int. J. Multiphase Flow 105, 159 (2018).

[25] M. Banowski, U. Hampel, E. Krepper, M. Beyer, and D. Lucas, Experimental investigation of two-phase pipe flow with ultrafast X-ray tomography and comparison with state-of-the-art CFD simulations, Nucl. Eng. Des. 336, 90 (2017).

[26] F. Fischer, D. Hoppe, E. Schleicher, G. Mattausch, H. Flaske, R. Bartel, and U. Hampel, An ultra fast electron beam X-ray tomography scanner, Meas. Sci. Technol. 19, 094002 (2008).

[27] F. Barthel, M. Bieberle, D. Hoppe, M. Banowski, and U. Hampel, Velocity measurement for two-phase flows based on ultrafast X-ray tomography, Flow Meas. Instrum. 46, 196 (2015).

[28] V. Dzelme, M. Sarma, V. Gea, M. Ščepanskis, and A. Jakovics, Transition of different vortical structures in rotating permanent magnets agitated flow: numerical and experimental neutron radiography investigation, in Proceedings of the 10th PAMIR International Conference on Fundamental and Applied MHD: DIEE, University of Cagliari, June 20-24, 2016, Cagliari, Italy (PAMIR Centre, 2016).

[29] M. Ščepanskis, M. Sarma, P. Vontobel, P. Trtik, K. Thomsen, A. Jakovics, and T. Beinerts, Assessment of electromagnetic stirrer agitated liquid metal flows by dynamic neutron radiography, Metall. Mater. Trans. B 48, 1045 (2017).

[30] R. Zboray and P. Trtik, 800 fps neutron radiography of air-water two-phase flow, MethodsX 5, 96 (2018).

[31] R. Zboray, R. Adams, M. Cortesi, and H.-M. Prasser, Development of a fast neutron imaging system for investigating two-phase flows in nuclear thermal-hydraulic phenomena: A status report, Nucl. Eng. Des. 273, 10 (2014).

[32] R. Zboray and P. Trtik, In-depth analysis of high-speed, cold neutron imaging of air-water two-phase flows, Flow Meas. Instrum. 66, 182 (2019).

[33] S. Heitkam, M. Rudolph, T. Lappan, M. Sarma, S. Eckert, P. Trtik, E. Lehmann, P. Vontobel, and K. Eckert, Neutron imaging of froth structure and particle motion, Miner. Eng. 119, 126 (2018).

[34] S. Heitkam, T. Lappan, S. Eckert, P. Trtik, and K. Eckert, Tracking of particles in froth using neutron imaging, Chem. Ing. Tech. 91, 1001 (2019).

[35] Y. Saito, K. Mishima, Y. Tobita, T. Suzuki, and M. Matsubayashi, Measurements of liquid-metal twophase flow by using neutron radiography and electrical conductivity probe, Exp. Therm. Fluid Sci. 29, 323 (2005), special issue, Third European-Japanese Two-Phase Flow Group Meeting.

[36] Y. Saito, K. Mishima, Y. Tobita, T. Suzuki, M. Matsubayashi, I. Lim, and J. Cha, Application of high frame-rate neutron radiography to liquid-metal two-phase flow research, Nucl. Instrum. Methods Phys. Res., Sect. A 542, 168 (2005). 
[37] M. Birjukovs, V. Dzelme, A. Jakovics, K. Thomsen, and P. Trtik, Argon bubble flow in liquid gallium in external magnetic field, Int. J. Appl. Electromagnet. Mech. (2020), doi:10.3233/JAE-209116.

[38] E. Lehmann, P. Vontobel, and L. Wiezel, Properties of the radiography facility NEUTRA at SINQ and its use as European reference facility, Nondestr. Test. Eval. 16, 191 (2001).

[39] R. Clift, J. Grace, and M. Weber, Bubbles, Drops, and Particles (Academic, New York, 1978). 